\title{
Effect of human faecal inoculum on in vitro fermentation variables
}

\author{
BY M. I. MCBURNEY AND L. U. THOMPSON \\ Department of Nutritional Sciences, University of Toronto, Toronto, \\ Ontario M5S 1A8, Canada
}

(Received 29 September 1986 - Accepted 19 March 1987)

\begin{abstract}
1. Homogenized and diluted faeces $(66 \cdot 6 \mathrm{~g} / 1)$ collected from one human source on three different months was incubated with four standard substrates (oat bran, wheat bran, red kidney beans (Phaseolus vulgaris) and guar gum) for $4,8,12$ and $24 \mathrm{~h}$.

2. Neutral-detergent fibre and organic matter $(\mathrm{OM})$ digestibility measurements and gas production (ml gas/g $\mathrm{OM}$ ) were influenced by substrate and incubation period but not by day of collection.

3. Production of short-chain fatty acids (SCFA) (mmol/g OM) was a function of substrate and incubation period but not day of collection at 4,8 and $12 \mathrm{~h}$. Rapidly fermentable substrates such as red kidney beans and guar gum did not ferment beyond $12 \mathrm{~h}$ and SCFA values were not different at $24 \mathrm{~h}$.

4. Substrates differed in amount, rate and type of SCFA produced.

5. The results indicate that human faeces collected on different occasions were sufficiently uniform to yield similar in vitro fermentation findings among collections. Therefore, it is concluded that human faecal material is a practical source of micro-organisms to evaluate the fermentation properties of substrates.
\end{abstract}

The fermentation of dietary fibre and starch by anaerobic micro-organisms in the colon produces short-chain fatty acids (SCFA) (Cummings, 1983; Flourie et al. 1986) which are absorbed (Demigne \& Remesy, 1985) and may be physiologically important (Hoverstad, 1986). It has been demonstrated that fibre sources differ in rate and extent of fermentation (Mertens, 1973; McBurney et al. 1986) but it is difficult and expensive to measure their impact on SCFA production in vivo.

In vitro fermentation systems offer several benefits such as reduced costs, relative freedom from animal experimentation and, most importantly, the fact that the duration and conditions of fermentation can be controlled. Obviously, it is essential that microbial species representative of the human colon be used in in vitro fermentations if the information gained is to be useful in understanding the role of the colonic fermentation in human nutrition and health. The most accessible and non-invasive means to obtain such a population of micro-organisms is from freshly defaecated human faeces. Since total bacterial counts, numbers of species and the distribution of species have not been found to change significantly within the colon of healthy subjects given bran (Drasar et al. 1976; Fuchs et al. 1976), bagasse (Walters et al. 1975), guar gum (Drasar \& Jenkins, 1976) and pectin (Doyle et al. 1981) when compared with a low-fibre control, it is assumed that human faeces are an adequate source of micro-organisms. Furthermore, there do not appear to be any detectable regional differences within the colon (Moore et al. 1978) of sudden-death victims and the flora of the mucosa is as complex as that of faeces (Croucher et al. 1983). Consequently, human faecal micro-organisms have been used in several in vitro studies pertaining to colonic fermentation (Miller \& Wolin, 1981; Perman et al. 1981; Edwards et al. 1985; McBurney et al. 1985; Englyst \& Macfarlane, 1986; Macfarlane \& Englyst, 1986; Macfarlane et al. 1986; Tomlin et al. 1986) yet it is unknown if human faecal material collected on different occasions to be used as an inoculum source will yield similar fermentation findings. The objectives of the present study were: (1) to determine if fermentation variables of four standard substrates changed when micro-organisms were collected from faecal material of a single individual on three different occasions; (2) to determine if an in vitro batch fermentation system could be used to measure SCFA. 
EXPERIMENTAL

Materials

Four whole foods were chosen to be the standards. They were: American Association of Cereal Chemists wheat bran, guar gum, oat bran and red kidney beans (Phaseolus vulgaris). These foods were chosen for their range of fermentabilities and fibre types.

The wheat bran, oat bran, and red kidney beans were ground through a $0.5 \mathrm{~mm}$ screen in a cyclone mill. Portions were analysed for dry matter and organic matter (OM) contents of each sample in duplicate by drying overnight at $100^{\circ}$, weighing and then ashing. Protein and fat contents were measured by standard methods of the Association of Official Analytical Chemists (1984) and total carbohydrate was estimated by difference. Total dietary fibre (TDF) content was estimated by the method of Prosky et al. (1986). Available carbohydrate was calculated by difference between the total carbohydrate and TDF. The neutral-detergent fibre (NDF) content was determined by the modified detergent method (Robertson \& Van Soest, 1982); the amylase solution (A 6880; Sigma Chemical Co, St Louis, Mo.) was replaced by $0.25 \mathrm{ml}$ Termamyl enzyme, a heat stable $\alpha$-amylase $120 \mathrm{~L}$ (Novo Laboratories, Copenhagen, Denmark). The TDF method (Prosky et al.) measures both the water-soluble and insoluble unavailable carbohydrate and non-carbohydrate components whereas the NDF procedure (Robertson \& Van Soest, 1982) measures the insoluble unavailable carbohydrate and non-carbohydrate components. However, it must be recognized that all the nitrogen measured in the fibre residue of the TDF method is assumed to be true protein. Therefore the $\mathrm{N}$ content of the fibre residue is multiplied by 6.25 (assuming $16 \% \mathrm{~N}$ in proteins) and this amount of protein is subtracted from the fibre residue to determine the TDF of the food. Consequently, the insoluble unavailable noncarbohydrate and therefore the TDF content of foods which contain significant amounts of $\mathrm{N}$ in the form of lignin or Maillard products would be underestimated. The NDF method, however, includes the $\mathbf{N}$ fraction arising from lignin and Maillard products as part of the insoluble dietary fibre matrix. Consequently, the difference between TDF and NDF is only a crude estimate of the soluble unavailable carbohydrate content of foods. Compositional data are presented in Table 1.

\section{Fermentation methods}

The batch-culture technique utilizing human faecal microflora (McBurney et al. 1985) was used with two replicate samples being run for each sample at each time. A $0.5 \mathrm{~g}$ sample was weighed into a $100 \mathrm{ml}$ serum bottle. The fermentation medium contained $(/ 1): 2.49 \mathrm{~g}$ trypticase peptone, $1.00 \mathrm{~g}$ ammonium bicarbonate, $8.75 \mathrm{~g}$ sodium bicarbonate, $1.43 \mathrm{~g}$ anhydrous sodium phosphate, $1.55 \mathrm{~g}$ anhydrous potassium phosphate monobasic, $0.60 \mathrm{~g}$ magnesium sulphate, $0.12 \mathrm{mg}$ resazurin, $1.12 \mathrm{mmol}$ calcium chloride, $0.63 \mathrm{mmol}$ manganous chloride, $0.15 \mathrm{mmol}$ cobalt chloride, $0.04 \mathrm{mmol}$ ferric chloride. Medium $(40 \mathrm{ml})$ was added to the samples approximately $12-24 \mathrm{~h}$ before the start of the incubation so that the samples would be hydrated when the inoculum was added. The serum bottle contents were then reduced using the conditions and reducing agents described by Goering \& Van Soest (1970), sealed with a butyl rubber stopper with crimped metal seal (Miller \& Wolin, 1974) and stored in the refrigerator overnight to limit the possibility of microbial growth. At 1-2 $\mathrm{h}$ before inoculation the bottles were put into a $37^{\circ}$ water-bath.

Fresh human faeces were collected on three separate occasions from a healthy individual who had been eating an unspecified Western diet and had not taken antibiotics for 1 year or more. The faecal stool was collected into a tared blender containing $400 \mathrm{ml}$ distilled water and reducing solution $(15: 2, \mathrm{v} / \mathrm{v})$ which had been warmed to $37^{\circ}$. The faeces were further diluted $(66.6 \mathrm{~g} / 1)$, blended for $30 \mathrm{~s}$ to detach cellulolytic micro-organisms which might be associated with the faecal fibre matrix, and then squeezed through a $41 \mu \mathrm{m}$ Nitex 
Table 1. Composition of the food samples ( $\mathrm{g} / \mathrm{kg}$ dry matter (DM))

\begin{tabular}{|c|c|c|c|c|c|c|c|c|}
\hline Sample & $\begin{array}{c}\mathrm{DM} \\
(\mathrm{g} / \mathrm{kg})\end{array}$ & Ash & Prot. & Fat & $\begin{array}{l}\text { Tot. } \\
\text { CHO }\end{array}$ & $\begin{array}{l}\text { Avail. } \\
\text { CHO }\end{array}$ & DF & NDF \\
\hline Oat bran & $91 \cdot 3$ & $3 \cdot 8$ & $18 \cdot 3$ & $7 \cdot 1$ & $70 \cdot 7$ & $52 \cdot 8$ & 17.9 & $11 \cdot 1$ \\
\hline $\begin{array}{l}\text { Red kidney bean } \\
\text { (Phaseolus vulgaris) }\end{array}$ & $89 \cdot 8$ & $3 \cdot 4$ & 24.9 & $1 \cdot 2$ & 70.5 & $50 \cdot 9$ & 19.6 & 16.7 \\
\hline Wheat bran & $93 \cdot 0$ & $7 \cdot 0$ & $15 \cdot 4$ & 1.9 & $75 \cdot 7$ & $31 \cdot 5$ & $44 \cdot 1$ & $41 \cdot 1$ \\
\hline Guar gum & $77 \cdot 4$ & $7 \cdot 1$ & 3.5 & 0.0 & $89 \cdot 4$ & 0.0 & $89 \cdot 4$ & 22.6 \\
\hline
\end{tabular}

Prot., protein; Tot. CHO, total carbohydrate; Avail. CHO, available carbohydrate, DF, dietary fibre; NDF, neutral-detergent fibre.

membrane and filtered through glass wool to remove fibre particulates (Jeraci, 1981). A portion $(10 \mathrm{ml})$ of this inoculum was injected by syringe through the septum of the serum bottles. At all times, faecal contents and containers were kept under a constant flow of carbon dioxide. Serum bottles were swirled at regular intervals. Samples were removed for analysis at $0,4,8,12$ and $24 \mathrm{~h}$.

Gas measurements were taken immediately on removal of the serum bottle from the water-bath using a modification of the method described by Miller \& Wolin (1974). A $60 \mathrm{ml}$ syringe with a three-way stopcock and needle was inserted into the bottle and $15 \mathrm{ml}$ gas were removed. The stopcock was then turned to isolate the syringe. The syringe was allowed to expand to ambient pressure and the volume of the contents was recorded. The amount of gas produced during the fermentation was calculated using Boyle's Law $\left(P_{1} V_{2}\right.$ $=P_{2} V_{1}$, where $P_{1}, P_{2}$ are pressures and $V_{1}, V_{2}$ are volumes of gas). The exact equation used to determine the amount of gas which had been produced was:

$$
V_{x}=4 \cdot 2 V_{3}-48,
$$

where $V_{x}(\mathrm{ml})$ is the volume of gas produced in the $48 \mathrm{ml}$ head volume of the serum bottle, $V_{3}$ is the volume $(\mathrm{ml})$ that the $15 \mathrm{ml}$ gas in the syringe occupied under conditions of ambient pressure and constant temperature. $V_{x}$ was then expressed as $\mathrm{ml}$ gas produced/g OM.

The serum bottles were then opened and $1 \mathrm{ml}$ copper sulphate solution $(10 \mathrm{~g} / \mathrm{l})$ was added to kill the micro-organisms. A $1.5 \mathrm{ml}$ portion was taken from each flask, filtered through a $0.22 \mu \mathrm{m}$ Millipore filter to remove any bacterial and fibrous particulate matter, and analysed for SCFA by high-performance liquid chromatography using a modification of the method of Mayer (1986). Filtrate $(30 \mu \mathrm{l})$ was injected directly on to a BioRad organic acid column HPX-87H kept at $37^{\circ}$ and absorbance was measured at $214 \mathrm{~nm}$. The eluant was $0.003 \mathrm{M}$-sulphuric acid at a flow rate of $0.6 \mathrm{ml} / \mathrm{min} . \mathrm{C}_{1}-\mathrm{C}_{7}$ SCFA standards were obtained from Supelco.

The remaining contents of the serum bottles were then quantitatively transferred to 600 $\mathrm{ml}$ Berzelius beakers and refluxed with $100 \mathrm{ml}$ neutral-detergent solution for $1 \mathrm{~h}$ at $100^{\circ}$ (Robertson \& Van Soest, 1982). The neutral-detergent residues were dried overnight at $100^{\circ}$ and weighed. The NDF method extracts endogenously secreted mucus and bacterially synthesized exopolysaccharides in order to recover undigested cellulose, hemicellulose and lignin (Robertson \& Van Soest, 1982; Marlett \& Johnson, 1985); therefore, it is possible to calculate the true digestibility of the NDF fraction. However, since the inoculum does contribute some additional OM and NDF, even though the faeces are filtered, the digestibility calculations of $\mathrm{OM}$ and NDF are adjusted as follows:

$$
\text { digestibility }=\frac{\text { original weight }- \text { (residue weight }-0 \mathrm{~h} \text { inoculum weight) }}{\text { original weight }} \text {. }
$$


Table 2. Digestibilities of neutral-detergent fibre of four substrates measured in vitro using human faecal inoculum collected from one individual on three different days

(Mean values with their standard errors)

\begin{tabular}{|c|c|c|c|c|c|c|c|}
\hline \multirow[b]{3}{*}{ Substrate } & \multirow{3}{*}{$\begin{array}{l}\text { Incubation } \\
\text { period } \\
\text { (h) }\end{array}$} & \multicolumn{6}{|c|}{ Day of collection } \\
\hline & & \multicolumn{2}{|c|}{1} & \multicolumn{2}{|c|}{2} & \multicolumn{2}{|c|}{3} \\
\hline & & Mean & $\mathbf{S E}$ & Mean & SE & Mean & $S E$ \\
\hline Oat bran & $\begin{array}{r}4 \\
8 \\
12 \\
24\end{array}$ & $\begin{array}{l}0.528 \\
0 \cdot 807 \\
0 \cdot 813 \\
0.873\end{array}$ & $\begin{array}{l}0.035 \\
0.014 \\
0.023 \\
0.019\end{array}$ & $\begin{array}{l}0.500 \\
0.734 \\
0.906 \\
0.929\end{array}$ & $\begin{array}{l}0.004 \\
0.026 \\
0.022 \\
0.016\end{array}$ & $\begin{array}{l}0.479 \\
0.626 \\
0.830 \\
0.904\end{array}$ & $\begin{array}{l}0.028 \\
0.009 \\
0.001 \\
0.001\end{array}$ \\
\hline Wheat bran & $\begin{array}{r}4 \\
8 \\
12 \\
24\end{array}$ & $\begin{array}{l}0 \cdot 166 \\
0 \cdot 334 \\
0 \cdot 396 \\
0 \cdot 440\end{array}$ & $\begin{array}{l}0.002 \\
0.006 \\
0.004 \\
0.001\end{array}$ & $\begin{array}{l}0.146 \\
0.308 \\
0.400 \\
0.467\end{array}$ & $\begin{array}{l}0.006 \\
0.002 \\
0.009 \\
0.011\end{array}$ & $\begin{array}{l}0.180 \\
0.292 \\
0.394 \\
0.437\end{array}$ & $\begin{array}{l}0.018 \\
0.001 \\
0.030 \\
0.001\end{array}$ \\
\hline $\begin{array}{l}\text { Red kidney bean } \\
\text { (Phaseolus vulgaris) }\end{array}$ & $\begin{array}{r}4 \\
8 \\
12 \\
24\end{array}$ & $\begin{array}{l}0.356 \\
0.567 \\
0.635 \\
0.659\end{array}$ & $\begin{array}{l}0.012 \\
0.064 \\
0.022 \\
0.012\end{array}$ & $\begin{array}{l}0.391 \\
0.638 \\
0.711 \\
0.656\end{array}$ & $\begin{array}{l}0.003 \\
0.048 \\
0.008 \\
0.045\end{array}$ & $\begin{array}{l}0.422 \\
0.599 \\
0.715 \\
0.792\end{array}$ & $\begin{array}{l}0.018 \\
0.008 \\
0.011 \\
0.008\end{array}$ \\
\hline Guar gum & $\begin{array}{r}4 \\
8 \\
12 \\
24\end{array}$ & $\begin{array}{l}0 \cdot 830 \\
0 \cdot 803 \\
0.922 \\
0.960\end{array}$ & $\begin{array}{l}0.007 \\
0.106 \\
0.031 \\
0.019\end{array}$ & $\begin{array}{l}0.763 \\
0.942 \\
0.903 \\
0.951\end{array}$ & $\begin{array}{l}0.139 \\
0.036 \\
0.097 \\
0.037\end{array}$ & $\begin{array}{l}0.790 \\
0.865 \\
1.000 \\
1.000\end{array}$ & $\begin{array}{l}0.021 \\
0.021 \\
0.000 \\
0.000\end{array}$ \\
\hline
\end{tabular}

Analysis of variance

$\begin{array}{lcc}\text { Source } & \text { df } & \begin{array}{c}\text { Statistical significance } \\ P<\end{array} \\ \text { Collection day (C) } & & \text { NS } \\ \text { Sample (S) } & 2 & 0.001 \\ \text { Incubation period (I) } & 3 & 0.001 \\ \mathrm{C} \times \mathrm{S} & 3 & \mathrm{NS} \\ \mathrm{C} \times \mathrm{I} & 6 & \mathrm{NS} \\ \mathrm{S} \times \mathrm{I} & 6 & 0.001\end{array}$

NS, Not significant.

Statistics

Results were analysed by analysis of variance using a SPSS PC + statistical package on a microcomputer.

\section{RESULTS}

The wet faeces had a mean weight of 125 (SE 47) $\mathrm{g}$. Table 2 shows the NDF digestibilities. The effect of day of collection was not significant whereas sample and incubation period were significant, as expected. Similar results were observed for OM digestibilites (Table 3). It is important to note that all samples except guar gum had significant amounts of protein and available carbohydrate which was fermented within the first $4 \mathrm{~h}$ and produced $\mathrm{OM}$ digestibilities ranging from 0.65 to 0.95 . The smaller increase in OM digestibility from 4 to $24 \mathrm{~h}$ reflected both the amount of NDF in these şamples and its fermentation rate. NDF and $\mathrm{OM}$ digestibilities approached the maximum extent of digestion by $12 \mathrm{~h}$ for both guar gum and red kidney beans whereas the slower fermenting wheat bran with its larger NDF content continued to be consumed by micro-organisms for the entire $24 \mathrm{~h}$. Total gas 
Table 3. Digestibilities of organic matter of four substrates measured in vitro using human faecal inoculum collected from one individual on three different days

(Mean values with their standard errors)

\begin{tabular}{|c|c|c|c|c|c|c|c|}
\hline \multirow[b]{3}{*}{ Substrate } & \multirow{3}{*}{$\begin{array}{l}\text { Incubation } \\
\text { period } \\
\text { (h) }\end{array}$} & \multicolumn{6}{|c|}{ Day of collection } \\
\hline & & \multicolumn{2}{|c|}{1} & \multicolumn{2}{|c|}{2} & \multicolumn{2}{|c|}{3} \\
\hline & & Mean & SE & Mean & SE & Mean & SE \\
\hline Oat bran & $\begin{array}{r}4 \\
8 \\
12 \\
24\end{array}$ & $\begin{array}{l}0.948 \\
0.979 \\
0.979 \\
0.986\end{array}$ & $\begin{array}{l}0.004 \\
0.001 \\
0.003 \\
0.002\end{array}$ & $\begin{array}{l}0.945 \\
0.971 \\
0.990 \\
0.992\end{array}$ & $\begin{array}{l}0.001 \\
0.003 \\
0.002 \\
0.002\end{array}$ & $\begin{array}{l}0.942 \\
0.962 \\
0.981 \\
0.989\end{array}$ & $\begin{array}{l}0.003 \\
0.001 \\
0.001 \\
0.001\end{array}$ \\
\hline Wheat bran & $\begin{array}{r}4 \\
8 \\
12 \\
24\end{array}$ & $\begin{array}{l}0.686 \\
0.727 \\
0.752 \\
0.772\end{array}$ & $\begin{array}{l}0.028 \\
0.002 \\
0.001 \\
0.001\end{array}$ & $\begin{array}{l}0.650 \\
0.716 \\
0.754 \\
0.781\end{array}$ & $\begin{array}{l}0.003 \\
0.001 \\
0.004 \\
0.004\end{array}$ & $\begin{array}{l}0.663 \\
0.710 \\
0.751 \\
0.769\end{array}$ & $\begin{array}{l}0.008 \\
0.001 \\
0.001 \\
0.001\end{array}$ \\
\hline $\begin{array}{l}\text { Red kidney bean } \\
\text { (Phaseolus vulgaris) }\end{array}$ & $\begin{array}{r}4 \\
8 \\
12 \\
24\end{array}$ & $\begin{array}{l}0.928 \\
0.936 \\
0.946 \\
0.949\end{array}$ & $\begin{array}{l}0.022 \\
0.009 \\
0.004 \\
0.002\end{array}$ & $\begin{array}{l}0.900 \\
0.946 \\
0.957 \\
0.949\end{array}$ & $\begin{array}{l}0.014 \\
0.007 \\
0.001 \\
0.007\end{array}$ & $\begin{array}{l}0.914 \\
0.941 \\
0.948 \\
0.969\end{array}$ & $\begin{array}{l}0.004 \\
0.001 \\
0.001 \\
0.001\end{array}$ \\
\hline Guar gum & $\begin{array}{r}4 \\
8 \\
12 \\
24\end{array}$ & $\begin{array}{l}0.962 \\
0.956 \\
0.982 \\
0.991\end{array}$ & $\begin{array}{l}0.001 \\
0.024 \\
0.007 \\
0.004\end{array}$ & $\begin{array}{l}0.947 \\
0.987 \\
0.978 \\
0.989\end{array}$ & $\begin{array}{l}0.032 \\
0.008 \\
0.022 \\
0.008\end{array}$ & $\begin{array}{l}0.953 \\
0.970 \\
1.000 \\
1.000\end{array}$ & $\begin{array}{l}0.004 \\
0.005 \\
0.001 \\
0.001\end{array}$ \\
\hline \multicolumn{8}{|c|}{ Analysis of variance } \\
\hline \multicolumn{2}{|r|}{ Source } & & df & \multicolumn{2}{|c|}{$\begin{array}{c}\text { Statistical significance } \\
P<\end{array}$} & & \\
\hline & \multicolumn{2}{|l|}{$\begin{array}{l}\text { Collection day (C) } \\
\text { Sample (S) } \\
\text { Incubation period (I) }\end{array}$} & $\begin{array}{l}2 \\
3 \\
3\end{array}$ & & & & \\
\hline & $\begin{array}{l}\mathrm{C} \times \mathrm{S} \\
\mathrm{C} \times \mathrm{I} \\
\mathrm{S} \times \mathrm{I}\end{array}$ & & $\begin{array}{l}6 \\
6 \\
6\end{array}$ & \multicolumn{2}{|c|}{$\begin{array}{l}0.05 \\
0.001\end{array}$} & & \\
\hline
\end{tabular}

NS, Not significant.

production ( $\mathrm{ml}$ gas/g OM) differed significantly with sample and fermentation time (incubation period) but not with day of collection (Table 4). The total SCFA (mmol produced/g OM) are presented in Table 5 with the analysis of variance values for results at 4,8 and $12 \mathrm{~h}$. The effect of day of collection was not significant for the 4,8 and $12 \mathrm{~h}$ times but there was a day-of-collection effect at $24 \mathrm{~h}$. However, two of the four substrates, guar gum and red kidney beans, had reached the extent of fermentation by $12 \mathrm{~h}$ (Table 2 and 3 ) and there was virtually no fermentable NDF or OM available from 12 to $24 \mathrm{~h}$. Consequently, a non-significant increase in digestibility was observed beyond $12 \mathrm{~h}$. Therefore, further SCFA production for these two substrates had to be a function of microbial lysis and refermentation. Paired $t$ tests of the SCFA produced (mmol/g OM) at $12 \mathrm{~h} \mathrm{v} .24 \mathrm{~h}$ for the guar gum and red kidney bean samples confirmed that the means were not different $(P>0.05)$ whereas significantly more SCFA had been produced from 12 to $24 \mathrm{~h}$ for oat bran $(P<0.01)$ and wheat bran $(P<0.05)$.

Table 6 shows the average mmol SCFA produced/g OM from the three collections as 
Table 4. Gas production ( $\mathrm{ml} / \mathrm{g}$ organic matter) measured in vitro on four substrates using human faecal inoculum collected from one individual on three different days

(Mean values with their standard errors)

\begin{tabular}{|c|c|c|c|c|c|c|c|}
\hline \multirow[b]{3}{*}{ Substrate } & \multirow{3}{*}{$\begin{array}{l}\text { Incubation } \\
\text { period } \\
\text { (h) }\end{array}$} & \multicolumn{6}{|c|}{ Day of collection } \\
\hline & & \multicolumn{2}{|c|}{1} & \multicolumn{2}{|c|}{2} & \multicolumn{2}{|c|}{3} \\
\hline & & Mean & $\mathrm{SE}$ & Mean & SE & Mean & SE \\
\hline Oat bran & $\begin{array}{r}4 \\
8 \\
12 \\
24\end{array}$ & $\begin{array}{r}60 \cdot 3 \\
110 \cdot 6 \\
112.7 \\
115.0\end{array}$ & $\begin{array}{l}4 \cdot 5 \\
4 \cdot 7 \\
1 \cdot 9 \\
9 \cdot 8\end{array}$ & $\begin{array}{r}41.8 \\
87.8 \\
107.7 \\
117.7\end{array}$ & $\begin{array}{l}0 \cdot 1 \\
4 \cdot 5 \\
6 \cdot 7 \\
2 \cdot 4\end{array}$ & $\begin{array}{r}42 \cdot 0 \\
92 \cdot 6 \\
101 \cdot 8 \\
117 \cdot 9\end{array}$ & $\begin{array}{l}0 \cdot 1 \\
0 \cdot 1 \\
9 \cdot 3 \\
2 \cdot 3\end{array}$ \\
\hline Wheat bran & $\begin{array}{r}4 \\
8 \\
12 \\
24\end{array}$ & $\begin{array}{l}41 \cdot 0 \\
76 \cdot 7 \\
81 \cdot 2 \\
74 \cdot 1\end{array}$ & $\begin{array}{r}9 \cdot 0 \\
9 \cdot 0 \\
9 \cdot 3 \\
11 \cdot 2\end{array}$ & $\begin{array}{l}41 \cdot 0 \\
58 \cdot 7 \\
81 \cdot 2 \\
85 \cdot 9\end{array}$ & $\begin{array}{l}0 \cdot 1 \\
0 \cdot 1 \\
4 \cdot 5 \\
0 \cdot 1\end{array}$ & $\begin{array}{l}36 \cdot 6 \\
61 \cdot 4 \\
79 \cdot 4 \\
88 \cdot 3\end{array}$ & $\begin{array}{l}0 \cdot 1 \\
6 \cdot 8 \\
2 \cdot 2 \\
2 \cdot 2\end{array}$ \\
\hline $\begin{array}{l}\text { Red kidney bean } \\
\text { (Phaseolus vulgaris) }\end{array}$ & $\begin{array}{r}4 \\
8 \\
12 \\
24\end{array}$ & $\begin{array}{r}48 \cdot 1 \\
93 \cdot 8 \\
121 \cdot 9 \\
129 \cdot 6\end{array}$ & $\begin{array}{l}4 \cdot 6 \\
7 \cdot 2 \\
3 \cdot 0 \\
0 \cdot 1\end{array}$ & $\begin{array}{c}55 \cdot 3 \\
105 \cdot 2 \\
133 \cdot 3 \\
136 \cdot 2\end{array}$ & $\begin{array}{l}7 \cdot 1 \\
4 \cdot 9 \\
4 \cdot 9 \\
2 \cdot 5\end{array}$ & $\begin{array}{r}55 \cdot 7 \\
91 \cdot 5 \\
120 \cdot 2 \\
127 \cdot 3\end{array}$ & $\begin{array}{l}2 \cdot 3 \\
9 \cdot 5 \\
0 \cdot 1 \\
7 \cdot 1\end{array}$ \\
\hline Guar gum & $\begin{array}{r}4 \\
8 \\
12 \\
24\end{array}$ & $\begin{array}{r}0 \cdot 0 \\
101 \cdot 2 \\
113 \cdot 2 \\
120 \cdot 1\end{array}$ & $\begin{array}{l}0 \cdot 0 \\
0 \cdot 9 \\
3 \cdot 8 \\
3 \cdot 3\end{array}$ & $\begin{array}{r}0.0 \\
115.9 \\
109 \cdot 4 \\
152.4\end{array}$ & $\begin{array}{r}0 \cdot 0 \\
16 \cdot 2 \\
16 \cdot 2 \\
4 \cdot 3\end{array}$ & $\begin{array}{r}4 \cdot 5 \\
110 \cdot 3 \\
106 \cdot 3 \\
114 \cdot 3\end{array}$ & $\begin{array}{r}4.5 \\
7.8 \\
11 \cdot 7 \\
4.0\end{array}$ \\
\hline \multicolumn{8}{|c|}{ Analysis of variance } \\
\hline & Source & & df & \multicolumn{2}{|c|}{$\begin{array}{c}\text { Statistical significance } \\
P<\end{array}$} & & \\
\hline & $\begin{array}{l}\text { Collection day }(C) \\
\text { Sample }(S) \\
\text { Incubation period (I }\end{array}$ & & $\begin{array}{l}2 \\
3 \\
3\end{array}$ & \multicolumn{2}{|c|}{$\begin{array}{c}\text { NS } \\
0 \cdot 001 \\
0 \cdot 001\end{array}$} & & \\
\hline & $\begin{array}{l}\mathrm{C} \times \mathrm{S} \\
\mathrm{C} \times \mathrm{I} \\
\mathrm{S} \times \mathrm{I}\end{array}$ & & $\begin{array}{l}6 \\
6 \\
6\end{array}$ & \multicolumn{2}{|c|}{$\begin{array}{c}0.001 \\
\text { NS } \\
0.001\end{array}$} & & \\
\hline
\end{tabular}

NS, Not significant.

well as the contributions of the individual acids. Generally at all times, the amounts of organic acids produced were largest with guar gum $>$ red kidney beans, oat bran $>$ wheat bran. Red kidney beans and guar gum had much greater rates of SCFA production since the greater amount of SCFA produced were generated from 0 to $12 \mathrm{~h}$. Formic acid was produced in the early phases of all fermentations and then disappeared by $24 \mathrm{~h}$. This phenomenum was similarly observed by Miller \& Wolin (1981) and indicates that the balance between the micro-organisms which produce and those which use formic acid shifts during the fermentation. Production of acetic, propionic and butyric acids kept increasing as the period of incubation increased.

The molar ratios of acetic: propionic: butyric acid are presented in Table 7. Acetic acid was the primary SCFA produced but its molar ratio typically decreased with the period of incubation whereas the proportion of butyric acid increased. Guar gum produced significantly more propionic acid (4-24 h values) $(0 \cdot 247-0 \cdot 294)$ and less butyric acid $(0.137-0 \cdot 129)$ than any of the other substrates (Table 7). Molar ratios of other studies, in 
Table 5. Production of short-chain fatty acids (SCFA) (mmol SCFA/g organic matter) measured in vitro on four substrates using human faecal inoculum collected from one individual on three different days

(Mean values with their standard errors)

\begin{tabular}{|c|c|c|c|c|c|c|c|}
\hline \multirow[b]{3}{*}{ Substrate } & \multirow{3}{*}{$\begin{array}{l}\text { Incubation } \\
\text { period } \\
\text { (h) }\end{array}$} & \multicolumn{6}{|c|}{ Day of collection } \\
\hline & & \multicolumn{2}{|c|}{1} & \multicolumn{2}{|c|}{2} & \multicolumn{2}{|c|}{3} \\
\hline & & Mean & SE & Mean & SE & Mean & SE \\
\hline Oat bran & $\begin{array}{r}4 \\
8 \\
12 \\
24\end{array}$ & $\begin{array}{l}3 \cdot 8 \\
5 \cdot 6 \\
6 \cdot 6 \\
7 \cdot 1\end{array}$ & $\begin{array}{l}0 \cdot 2 \\
0 \cdot 4 \\
0 \cdot 1 \\
0 \cdot 1\end{array}$ & $\begin{array}{l}3 \cdot 5 \\
6 \cdot 0 \\
6 \cdot 9 \\
7 \cdot 4\end{array}$ & $\begin{array}{l}0 \cdot 1 \\
0 \cdot 2 \\
0 \cdot 2 \\
0 \cdot 1\end{array}$ & $\begin{array}{l}3 \cdot 2 \\
6 \cdot 0 \\
6 \cdot 4 \\
7 \cdot 0\end{array}$ & $\begin{array}{l}0 \cdot 1 \\
0.1 \\
0 \cdot 2 \\
0 \cdot 1\end{array}$ \\
\hline Wheat bran & $\begin{array}{r}4 \\
8 \\
12 \\
24\end{array}$ & $\begin{array}{l}3 \cdot 4 \\
5 \cdot 0 \\
5 \cdot 4 \\
5 \cdot 9\end{array}$ & $\begin{array}{l}0 \cdot 1 \\
0 \cdot 1 \\
0 \cdot 0 \\
0 \cdot 1\end{array}$ & $\begin{array}{l}4 \cdot 1 \\
5 \cdot 2 \\
5 \cdot 9 \\
6 \cdot 7\end{array}$ & $\begin{array}{l}0 \cdot 1 \\
0 \cdot 1 \\
0 \cdot 1 \\
0 \cdot 1\end{array}$ & $\begin{array}{l}3 \cdot 4 \\
4 \cdot 4 \\
5 \cdot 0 \\
5 \cdot 8\end{array}$ & $\begin{array}{l}0 \cdot 3 \\
0 \cdot 1 \\
0 \cdot 1 \\
0 \cdot 5\end{array}$ \\
\hline $\begin{array}{l}\text { Red kidney bean } \\
\text { (Phaseolus vulgaris) }\end{array}$ & $\begin{array}{r}4 \\
8 \\
12 \\
24\end{array}$ & $\begin{array}{l}3 \cdot 3 \\
5 \cdot 6 \\
6 \cdot 6 \\
7 \cdot 7\end{array}$ & $\begin{array}{l}0 \cdot 0 \\
0 \cdot 1 \\
0 \cdot 4 \\
0 \cdot 1\end{array}$ & $\begin{array}{l}3 \cdot 6 \\
6 \cdot 2 \\
7 \cdot 2 \\
7 \cdot 8\end{array}$ & $\begin{array}{l}0.0 \\
0.2 \\
0.2 \\
0.2\end{array}$ & $\begin{array}{l}3 \cdot 4 \\
6 \cdot 1 \\
7 \cdot 1 \\
6 \cdot 7\end{array}$ & $\begin{array}{l}0 \cdot 1 \\
0 \cdot 5 \\
0 \cdot 2 \\
0 \cdot 1\end{array}$ \\
\hline Guar gum & $\begin{array}{r}4 \\
8 \\
12 \\
24\end{array}$ & $\begin{array}{r}6 \cdot 2 \\
8 \cdot 9 \\
10 \cdot 3 \\
10 \cdot 2\end{array}$ & $\begin{array}{l}1 \cdot 0 \\
0 \cdot 1 \\
0 \cdot 2 \\
0 \cdot 1\end{array}$ & $\begin{array}{r}6.9 \\
8.9 \\
9.1 \\
11.3\end{array}$ & $\begin{array}{l}0.1 \\
0.7 \\
0.2 \\
0.1\end{array}$ & $\begin{array}{r}5 \cdot 6 \\
10 \cdot 4 \\
10 \cdot 7 \\
9 \cdot 3\end{array}$ & $\begin{array}{l}0 \cdot 1 \\
0 \cdot 8 \\
0 \cdot 3 \\
0 \cdot 2\end{array}$ \\
\hline \multicolumn{8}{|c|}{ Analysis of variance $(4,8,12 \mathrm{~h})$} \\
\hline \multicolumn{3}{|c|}{ Source } & df & \multicolumn{2}{|c|}{$\begin{array}{c}\text { Statistical significance } \\
P<\end{array}$} & & \\
\hline & \multicolumn{2}{|c|}{$\begin{array}{l}\text { Collection day (C) } \\
\text { Sample (S) } \\
\text { Incubation period (I) }\end{array}$} & $\begin{array}{l}2 \\
3 \\
3\end{array}$ & & & & \\
\hline & $\begin{array}{l}\mathrm{C} \times \mathrm{S} \\
\mathrm{C} \times \mathrm{I} \\
\mathrm{S} \times \mathrm{I} \\
\mathrm{C} \times \mathrm{S} \times \mathrm{I}\end{array}$ & & $\begin{array}{r}6 \\
6 \\
6 \\
18\end{array}$ & $\begin{array}{l}0 \\
1 \\
0 \\
0\end{array}$ & & & \\
\hline
\end{tabular}

NS, Not significant.

vivo and in vitro, are presented in Table 8 . The similarity of the results of the present study to in vivo measurements provides strong evidence that this in vitro system can be used to model the human colon.

\section{DISCUSSION}

NDF and OM digestibilities as well as gas production did not differ among collections. SCFA production was not affected by collection at 4,8 and $12 \mathrm{~h}$ incubation but it was at $24 \mathrm{~h}$. Since guar gum and red kidney beans had reached maximal digestion by $12 \mathrm{~h}$ and there was no further SCFA or gas production by $24 \mathrm{~h}$, it seems that the effect of day of collection at $24 \mathrm{~h}$ incubation may be a function of a dying fermentation and the balance of surviving microbial species. Obviously, the original inoculum establishes the range and viability of the micro-organisms present within the fermentation bottle. However, it 
Table 6. Total and individual short-chain fatty acid (SCFA) production (mmol SCFA/g organic matter) measured in vitro on four substrates using human faecal inoculum

(Mean values and their standard errors)

\begin{tabular}{|c|c|c|c|c|c|c|}
\hline Substrate & $\begin{array}{l}\text { Incubation } \\
\text { period } \\
\text { (h) }\end{array}$ & $\begin{array}{l}\text { Total } \\
\text { SCFA }\end{array}$ & $\begin{array}{l}\text { Formic } \\
\text { acid }\end{array}$ & $\begin{array}{l}\text { Acetic } \\
\text { acid }\end{array}$ & $\begin{array}{l}\text { Propionic } \\
\text { acid }\end{array}$ & $\begin{array}{l}\text { Butyric } \\
\text { acid }\end{array}$ \\
\hline Oat bran & $\begin{array}{r}4 \\
8 \\
12 \\
24\end{array}$ & $\begin{array}{l}3 \cdot 50 \\
5 \cdot 84 \\
6 \cdot 63 \\
7 \cdot 15\end{array}$ & $\begin{array}{l}0.22 \\
0.46 \\
0.60 \\
0.00\end{array}$ & $\begin{array}{l}1 \cdot 92 \\
2 \cdot 85 \\
3 \cdot 38 \\
3 \cdot 63\end{array}$ & $\begin{array}{l}0.62 \\
1.06 \\
1 \cdot 14 \\
1.33\end{array}$ & $\begin{array}{l}0.54 \\
1.06 \\
1.26 \\
1.44\end{array}$ \\
\hline Wheat bran & $\begin{array}{r}4 \\
8 \\
12 \\
24\end{array}$ & $\begin{array}{l}3 \cdot 62 \\
4 \cdot 86 \\
5 \cdot 41 \\
6 \cdot 10\end{array}$ & $\begin{array}{l}0.07 \\
0.10 \\
0.13 \\
0.05\end{array}$ & $\begin{array}{l}2 \cdot 16 \\
2 \cdot 75 \\
3 \cdot 04 \\
3 \cdot 35\end{array}$ & $\begin{array}{l}0.57 \\
0.77 \\
0.87 \\
1.05\end{array}$ & $\begin{array}{l}0.52 \\
0.79 \\
0.90 \\
1.07\end{array}$ \\
\hline $\begin{array}{l}\text { Red kidney bean } \\
\text { (Phaseolus vulgaris) }\end{array}$ & $\begin{array}{r}4 \\
8 \\
12 \\
24\end{array}$ & $\begin{array}{l}3.43 \\
5.96 \\
6 \cdot 98 \\
7 \cdot 37\end{array}$ & $\begin{array}{l}0.02 \\
0 \cdot 28 \\
0 \cdot 16 \\
0 \cdot 00\end{array}$ & $\begin{array}{l}2 \cdot 13 \\
3 \cdot 45 \\
4 \cdot 13 \\
4 \cdot 24\end{array}$ & $\begin{array}{l}0.53 \\
0.86 \\
0.98 \\
1 \cdot 12\end{array}$ & $\begin{array}{l}0.48 \\
0.97 \\
1 \cdot 17 \\
1.33\end{array}$ \\
\hline Guar gum & $\begin{array}{r}4 \\
8 \\
12 \\
24\end{array}$ & $\begin{array}{r}6 \cdot 24 \\
9 \cdot 37 \\
10 \cdot 01 \\
10 \cdot 27\end{array}$ & $\begin{array}{l}0.48 \\
0.72 \\
0.51 \\
0.00\end{array}$ & $\begin{array}{l}3 \cdot 31 \\
4 \cdot 72 \\
5 \cdot 08 \\
5 \cdot 35\end{array}$ & $\begin{array}{l}1 \cdot 35 \\
2 \cdot 25 \\
2 \cdot 61 \\
2 \cdot 74\end{array}$ & $\begin{array}{l}0 \cdot 73 \\
1.00 \\
1 \cdot 11 \\
1 \cdot 20\end{array}$ \\
\hline
\end{tabular}

Table 7. Fractional molar proportions of acetic, propionic and butyric acids measured in vitro on four substrates using human faecal inoculum collected from one individual on three different days

(Mean values with their standard errors)

\begin{tabular}{|c|c|c|c|c|c|c|c|}
\hline \multirow[b]{2}{*}{ Substrate } & \multirow{2}{*}{$\begin{array}{l}\text { Incubation } \\
\text { period } \\
\text { (h) }\end{array}$} & \multicolumn{2}{|c|}{ Acetic acid } & \multicolumn{2}{|c|}{ Propionic acid } & \multicolumn{2}{|c|}{ Butyric acid } \\
\hline & & Mean & $\mathrm{SE}$ & Mean & SE & Mean & SE \\
\hline Oat bran & $\begin{array}{r}4 \\
8 \\
12 \\
24\end{array}$ & $\begin{array}{l}0.620 \\
0.572 \\
0.585 \\
0.567\end{array}$ & $\begin{array}{l}0.022 \\
0.027 \\
0.016 \\
0.023\end{array}$ & $\begin{array}{l}0.201 \\
0.213 \\
0.198 \\
0.208\end{array}$ & $\begin{array}{l}0.009 \\
0 \cdot 016 \\
0 \cdot 013 \\
0 \cdot 010\end{array}$ & $\begin{array}{l}0 \cdot 179 \\
0 \cdot 215 \\
0 \cdot 217 \\
0 \cdot 225\end{array}$ & $\begin{array}{l}0.015 \\
0.020 \\
0.009 \\
0.014\end{array}$ \\
\hline Wheat bran & $\begin{array}{r}4 \\
8 \\
12 \\
24\end{array}$ & $\begin{array}{l}0.662 \\
0.639 \\
0.632 \\
0.614\end{array}$ & $\begin{array}{l}0.028 \\
0.010 \\
0.006 \\
0.010\end{array}$ & $\begin{array}{l}0.178 \\
0.178 \\
0.180 \\
0.191\end{array}$ & $\begin{array}{l}0.013 \\
0.009 \\
0.007 \\
0 \cdot 007\end{array}$ & $\begin{array}{l}0 \cdot 160 \\
0 \cdot 183 \\
0 \cdot 188 \\
0 \cdot 195\end{array}$ & $\begin{array}{l}0.130 \\
0.009 \\
0.004 \\
0.006\end{array}$ \\
\hline $\begin{array}{l}\text { Red kidney bean } \\
\text { (Phaseolus vulgaris) }\end{array}$ & $\begin{array}{r}4 \\
8 \\
12 \\
24\end{array}$ & $\begin{array}{l}0.678 \\
0.654 \\
0.658 \\
0.634\end{array}$ & $\begin{array}{l}0.021 \\
0.011 \\
0.014 \\
0.019\end{array}$ & $\begin{array}{l}0.169 \\
0.163 \\
0.156 \\
0.167\end{array}$ & $\begin{array}{l}0.015 \\
0 \cdot 013 \\
0 \cdot 008 \\
0 \cdot 010\end{array}$ & $\begin{array}{l}0 \cdot 153 \\
0 \cdot 184 \\
0 \cdot 186 \\
0 \cdot 199\end{array}$ & $\begin{array}{l}0.009 \\
0.004 \\
0.010 \\
0.011\end{array}$ \\
\hline Guar gum & $\begin{array}{r}4 \\
8 \\
12 \\
24\end{array}$ & $\begin{array}{l}0.614 \\
0.593 \\
0.578 \\
0.577\end{array}$ & $\begin{array}{l}0.008 \\
0.017 \\
0.009 \\
0.015\end{array}$ & $\begin{array}{l}0.247 \\
0.282 \\
0.296 \\
0.294\end{array}$ & $\begin{array}{l}0.014 \\
0.008 \\
0.012 \\
0.019\end{array}$ & $\begin{array}{l}0.137 \\
0.125 \\
0.126 \\
0.129\end{array}$ & $\begin{array}{l}0.021 \\
0.014 \\
0.011 \\
0.018\end{array}$ \\
\hline
\end{tabular}


Table 8. Fractional molar ratios of acetic (Ac), propionic ( $\mathrm{Pr}$ ), and butyric (Bu) acids in the caecum and faeces of man and other in vitro fermentation systems using human faecal material as an inoculum source

\begin{tabular}{lll}
\hline \multicolumn{1}{c}{ Site } & Ac:Pr:Bu & \multicolumn{1}{c}{ Reference } \\
\hline Caecum & $0 \cdot 73: 0 \cdot 17: 0 \cdot 10$ & Flourie et al. (1986) \\
Faecal dialysate & $0 \cdot 60: 0 \cdot 22: 0 \cdot 18$ & Rubenstein et al. (1969) \\
Faecal dialysate & $0 \cdot 57: 0 \cdot 28: 0 \cdot 15$ & Bjork et al. (1976) \\
Faecal dialysate & $0 \cdot 59: 0 \cdot 20: 0 \cdot 21$ & Cummings et al. (1979) \\
Whole stool & $0 \cdot 64: 0 \cdot 21: 0 \cdot 15$ & Spiller et al. (1980) \\
Whole stool & $0 \cdot 54: 0 \cdot 29: 0 \cdot 17$ & Fleming \& Rodriguez (1983) \\
Whole stool & $0 \cdot 65: 0 \cdot 19: 0 \cdot 16$ & Gray \& Olson (1985) \\
In vitro & & \\
Batch & $0 \cdot 42: 0 \cdot 21: 0 \cdot 37$ & Englyst \& Macfarlane (1986) \\
Batch & $0 \cdot 57: 0 \cdot 16: 0 \cdot 27$ & Macfarlane et al. (1986) \\
Batch & $0 \cdot 52: 0 \cdot 20: 0 \cdot 28$ & Macfarlane \& Englyst (1986) \\
Semi-continuous & $0 \cdot 69: 0 \cdot 24: 0 \cdot 06$ & Miller \& Wolin (1981) \\
Continuous & $0 \cdot 45: 0 \cdot 51: 0 \cdot 04$ & Edwards et al. (1985) \\
\hline \hline
\end{tabular}

appears that the substrate and its fermentability is the dominant influence on the fermentation profile until substrate becomes limiting. In this context, $12 \mathrm{~h}$ values should be used for comparison purposes since there would not be an effect of day of collection. However, the paired $t$ tests between the $12 \mathrm{~h}$ and $24 \mathrm{~h}$ SCFA levels for guar gum and red kidney beans demonstrated that despite the possibility of microbial lysis and refermentation the $24 \mathrm{~h}$ SCFA values had not significantly changed and could still be used to make physiologically important distinctions among foods. Furthermore, the average colonic retention is estimated to be approximately $24 \mathrm{~h}$ (Smith \& Eastwood, 1980; Krevsky et al. 1986) and it would be inappropriate to use incubation periods of less than $24 \mathrm{~h}$ to measure SCFA production of substrates such as wheat bran and oat bran which would still be fermenting within the colon.

The wheat bran, oat bran and kidney bean samples all contained significant amounts of available carbohydrate and protein which would produce SCFA and an in vitro fermentation. However, the microbial fermentation of guar gum which was approximately $900 \mathrm{~g}$ dietary fibre $/ \mathrm{kg}$ produced the largest quantities of SCFA. Not only were more SCFA produced from guar gum but they were produced within the first $12 \mathrm{~h}$, i.e. at a very rapid rate. Moreover, the fermentation of guar gum resulted in proportionally more propionate $(4-24 \mathrm{~h}$ values) $(0 \cdot 247-0 \cdot 294)$ and less butyrate $(0 \cdot 137-0 \cdot 129)$ then did the fermentation of other foods containing more protein and available carbohydrate and less dietary fibre. Oat bran had both the highest molar proportion of butyric acid and butyrate production level. It is apparent from the present study that both total SCFA production and the molar ratios of SCFA produced in a microbial fermentation can be influenced by substrate which could have significant nutritive and metabolic effects.

The role of the absorbed SCFA in human metabolism is not very well understood (Low, 1985; Pomare et al. 1985) but propionate may modify lipid and carbohydrate metabolism in the liver (Anderson \& Bridges, 1982; Chen et al. 1984) Butyrate and propionate have been suggested to be important energy sources for the colonic mucosa, thus promoting colonic health (Cummings, 1984). Butyrate may have antineoplastic properties since it has been shown to reduce the growth of human large-bowel cancer cells (Kim et al. 1981; Kruh, 1982).

If SCFA stimulate normal cell growth, as has been shown in ruminants (Flatt et al. 1959; 
Sander et al. 1959), one would expect increased cell proliferation at the site of greatest SCFA production. Therefore, cell proliferation and hyperplasia should be enhanced within the proximal colon on rapidly fermentable substrates such as guar gum, as seen in the present study, whereas more slowly fermenting materials like wheat bran might influence cell growth within the distal colon. Indeed, guar gum has been observed to cause greater hyperplasia in the proximal colon (Jacobs \& Lupton, 1984) while wheat bran produced the greater effect distally (Jacobs \& White, 1983). Certainly, the results from the present study indicate that substrate has a signifcant impact on the rate, amount and type of SCFA produced during microbial fermentation.

It is concluded that faecal material collected from humans at different times of the year provides a sufficiently uniform source of micro-organisms so that fermentation values such as digestibility estimates and gas or SCFA production can be compared between studies. Furthermore, a comparison of SCFA molar ratios with in vivo measurements indicates that in vitro results can be used to predict or interpret in vivo results.

This study was supported by grants from the Natural Sciences and Engineering Research Council of Canada and the National Health Research and Development Program of Canada. The authors would like to thank Dr T. Francis and Mr A. Mayer for their expert advice on the analysis of short-chain fatty acids by high-performance liquid chromatography, and Mrs F. Khan and Mr S. Mitchell for their technical assistance.

\section{REFERENCES}

Anderson, J. W. \& Bridges, S. R. (1982). American Journal of Clinical Nutrition 35, 840.

Association of Official Analytical Chemists (1984). Official Methods of Analysis, 14th ed, pp. 153-157 [S. Williams, editor]. Arlington: Association of Official Analytical Chemists, Inc.

Bjorke, J. T., Soergel, K. H. \& Wood, C. M. (1976). Gastroenterology 70, A/6/864.

Chen, W.-J., Anderson, J. W. \& Jennings, D. (1984). Proceedings of the Society for Experimental Biology and Medicine 175, 215-218.

Croucher, S. C., Houston, A. P., Bayliss, C. E. \& Turner R. J. (1983). Applied and Environmental Microbiology 45, 1025-1033.

Cummings, J. H. (1983). Lancet i, 1206-1209.

Cummings, J. H. (1984). Scandinavian Journal of Gastroenterology 19, 89-99.

Cummings, J. H., Hill, M. J., Bone, E. S., Branch, W. J. \& Jenkins, D. J. A. (1979). American Journal of Clinical Nutrition 32, 2094-2101.

Demigne, C. \& Remesy, C. (1985). Journal of Nutrition 115, 53-60.

Doyle, R. B., Wolfman, M., Varso, D. \& Floch, M. H. (1981). American Journal of Clinical Nutrition 34, 635.

Drasar, B. S. \& Jenkins, D. J. A. (1976). American Journal of Clinical Nutrition 29, 1410-1414.

Drasar, B. S., Jenkins, D. J. A. \& Cummings, J. H. (1976). Journal of Medical Microbiology 9, 423-431.

Edwards, C. A., Duerden, B. I. \& Read, N. W. (1985). Gastroenterology 88, 1903-1909.

Englyst, H. N. \& Macfarlane, G. T. (1986). Journal of the Science of Food and Agriculture 37, 699-706.

Fleming, S. E. \& Rodriguez, M. A. (1983). Journal of Nutrition 113, 1613-1625.

Flatt, W. P., Warner, R. G. \& Loosli, J. K. (1959). Journal of Dairy Science 41, 1593-1600.

Flourie, B., Florent, C., Jouany, J.-P., Thivend, P., Etanchaud, F. \& Rambaud, J.-C. (1986). Gastroenterology 19, $111-119$.

Fuchs, H.-M., Dorfman, S. \& Floch, M. H. (1976). American Journal of Clinical Nutrition 29, 1443-1447.

Goering, H. K. \& Van Soest, P. J. (1970). Forage Fiber Analysis (Apparatus, Reagents, Procedures and some Applications). Agriculture Handbook no. 379. Washington, DC: USDA.

Gray, G. \& Olson, A. C. (1985). Journal of Agricultural and Food Chemistry 33, 192-195.

Hoverstad, T. (1986). Scandinavian Journal of Gastroenterology 21, 257-260.

Jacobs, L. R. \& Lupton, J. R. (1984). American Journal of Physiology 246, G378-G385.

Jacobs, L. R. \& White, F. A. (1983). American Journal of Clinical Nutrition 37, 945-953.

Jeraci, J. L. (1981). Interactions between rumen and human fecal inocula and fiber substrates. MSc Thesis, Cornell University, Ithaca, New York.

Kim, Y. S., Tsao, D., Morita, A. \& Bella, A. (1981). In Colonic Carcinogenesis, pp. 317-323 [R. A. Malt and R. C. N. Williamson, editors]. Falk Symposium no. 31. Lancaster: MTP

Krevsky, B., Malmud, L. S., D'ecole, F., Maurer, A. H. \& Fisher, R. S. (1986). Gastroenterology 91, $1102-1112$. 
Kruh, J. (1982). Molecular and Cellular Biochemistry 42, 65-82.

Low, A. G. (1985). In Recent Advances of Animal Nutrition, pp. 87-111 [W. Haversig, editor]. London: Butterworths.

McBurney, M. I., Allen, M. S. \& Van Soest, P. J. (1986). Journal of the Science of Food and Agriculture 37, 666-672.

McBurney, M. I., Horvath, P. J., Jeraci, J. L. \& Van Soest, P. J. (1985). British Journal of Nutrition 53, $17-24$.

Macfarlane, G. T., Cummings, J. H. \& Allison, C. (1986). Journal of General Microbiology 132, 1647-1656.

Macfarlane, G. T. \& Englyst, H. N. (1986). Journal of Applied Bacteriology 60, 195-201.

Marlett, J. A. \& Johnson, E. J. (1985). Journal of Nutrition 115, 650-660.

Mayer, A. (1986). Physiological effects of colonically derived short chain fatty acids. MSc Thesis, University of Toronto, Toronto, Ontario.

Mertens, D. R. (1973). Application of theoretical mathematical models to cell wall digestion and forage intake in ruminants. PhD Thesis, Cornell University, Ithaca, New York.

Miller, T. L. \& Wolin, M. J. (1974). Applied Microbiology 27, 985-987.

Miller, T. L. \& Wolin, M. J. (1981). Applied and Environmental Microbiology 42, 400-407.

Moore, W. E. C., Cato, E. P. \& Holdeman, L. V. (1978). American Journal of Clinical Nutrition 31, S33-S42.

Perman, J. A., Modler, S. \& Olsen, A. C. (1981). Journal of Clinical Investigations 67, 643-650.

Pomare, E. W., Branch, W. J. \& Cummings, J. H. (1985). Journal of Clinical Investigations 75, $1448-1454$.

Prosky, L., Asp, N., Furda, I., DeVries, J. W., Schweizer, T. F. \& Harland, B. F. (1986). Journal of the Association of Official Analytical Chemists 68, 399.

Robertson, J. B. \& Van Soest, P. J. (1982). In The Analysis of Dietary Fiber in Food, pp 123-158 [W. P. T. James and $O$. Theander, editors]. New York: Marcel Dekker.

Rubenstein, R., Howard, A. V. \& Wrong, O. M. (1969). Clinical Sciences 37, 549-564.

Sander, E. G., Warner, R. S., Harrison, H. N. \& Loosli, J. K. (1959). Journal of Dairy Science 42, $1600-1605$.

Smith, A. N. \& Eastwood, M. A. (1980). In Medical Aspects of Dietary Fiber, pp. $27-42$ [G. A. Spiller and R. M. Kay, editors]. New York: Plenum Medical Book Co.

Spiller, G. A., Chernoff, M. C., Hill, R. A., Gates, J. E., Nassar, J. J. \& Shipley, E. A. (1980). American Journal of Clinical Nutrition 33, 754-759.

Tomlin, J., Read, N. W., Edwards, C. A. \& Duerden, B. I. (1986). British Journal of Nutrition 55, 481-486.

Walters, R. L., Baird, I. M., Davies, P. S., Hill, M. J., Drasar, B. S., Southgate, D. A. T., Green, J. \& Morgan, B. (1975). British Medical Journal ii, 536-538. 